\title{
FUZZY ANALYSIS OF COMFORT ALONG TRAVEL CHAINS
}

\author{
Lajos KISGYÖRGY1 , János TÓTH ${ }^{2}$ \\ ${ }^{1}$ Dept of Highway and Railway Engineering, Budapest University of Technology and Economics, Hungary \\ ${ }^{2}$ Dept of Transport Technology and Economics, Budapest University of Technology and Economics, Hungary
}

Received 5 July 2017; revised 23 January 2018, 13 June 2018; accepted 7 October 2018

\begin{abstract}
The competitiveness of a travel chain largely depends on the travel conditions along the sequence of journeys within the chain. This paper shows a method to analyse and to optimize the service quality along a travel chain. Travel comfort is a very important qualitative feature of the public transportation service, where travel comfort is used in a broader sense to describe ride quality and transfer quality including mobility, information, safety, security, and naturally comfort aspects. The analysis of travel comfort in the literature regards public transportation services. Several synthetic indices, which consider user judgment about service aspects, were developed to describe travel comfort, and comprehensive analyses have been published. However, to describe the competitiveness of the public transport the focus from the individual services should be moved toward the integrated service of the travel chain from the beginning to the end. The characteristics of travel comfort along the travel chain should be described and the location and rate of necessary interventions should be identified. In this paper we analyse the travel comfort features of travel chains. This paper proposes a method, which describes the travel comfort characteristics with synthetic indices based on the individual comfort indices of travel components, and uses a fuzzy approach to give an overall analysis of comfort conditions along the travel chain. The proposed method helps to identify the quality fluctuation and the weak points of a travel chain and makes the attractiveness of alternative travel chains comparable. An illustrative case study was carried out for one of the major transportation corridor of Budapest (Hungary), to exemplify the approach, where the validity of the method was tested as well. The results confirmed the usefulness and applicability of the methodology; by its application very valuable insights can be gained regarding the location and type of the necessary interventions. The results of our research are helpful to evaluate the actual service level of sustainable alternatives of individual car usage and to promote modal shift towards sustainable transportation modes.
\end{abstract}

Keywords: comfort analysis, travel chain, comfort index, fuzzy rules, public transportation, competitiveness.

\section{Introduction}

There are two interpretations of travel chains: the first interprets travel chain as a chain of daily trips, while the second sees them as the sequence of journeys within one trip. This paper uses the second interpretation, according to which travel chains are the integrated components of a trip. These components are: (1) walking trip at the beginning and the end of travel, (2) riding transportation vehicles or using these services, (3) transfers between rides. Travel chains and their analysis are essentials, because the users make their choice on among competing travel alternatives based on the attractiveness and competitiveness of the respective travel chains.

The attractiveness of travel chains, which influences competitiveness as well largely depends on the travel conditions along the chain (Kisgyörgy, Vasvári 2014). Improv- ing service quality is important for customizing habitual travellers and for attracting new users. The determination of these qualitative factors should be based on individual specific latent variables of environmental preferences, safety, security, comfort, convenience and flexibility. In this paper we refer to these individual specific latent variables collectively as travel comfort.

The evaluation systems of public transportation use quantitative and qualitative indicators. The aspects generally describing public transportation services can be distinguished into the characteristics that describe the service more properly (e.g., service frequency), and less easily measurable characteristics that depend more on the taste of the customer (e.g., comfort) (Eboli, Mazzulla 2009). The Transit Cooperative Research Program (TCRP) report

${ }^{*}$ Corresponding author. E-mail: toth.janos@mail.bme.hu 
(TRB 2003) defines a framework to determine the quality of service from the passenger's point of view, which is based on two factors, availability, and comfort and convenience. Availability determines if a public transportation service is an option for a particular trip; and comfort and convenience considered by the passengers describes the service quality (Iseki, Taylor 2010).

Determining public transportation service quality, recent research studies define different attributes, such as service scheduling and reliability, service coverage, information, comfort, cleanliness and safety and security (TRB 2003; Eboli, Mazzulla 2007, 2015; Tyrinopoulos, Antoniou 2008; Iseki, Taylor 2010; De Oña et al. 2013, 2014; De Oña, R., De Oña, J. 2015). Based on these studies, the service quality of transportation system depends on several factors, which can be classified in four topics: mobility, information, safety and security, and comfort.

Several studies dealt with the consequences of transfers on the service quality of public transportation; but mainly in the intermodal and multimodal context (Guihaire, Hao 2008; López-Lambas, Monzon 2010; Hernandez et al. 2016). Analysis of passengers' perception on transfers showed the importance of transfer quality on the perception on travel chains as a whole (Iseki, Taylor 2010; Dell'Olio et al. 2011; Cherry, Townsend 2012; De Abreu e Silva, Bazrafshan 2013; Cascetta, Cartenì 2014). However, integrated analysis of service quality along a travel chain was performed only for accessibility (Tsalis, Naniopoulos 2012).

However, research work on public transportation service quality does not offer a practical framework for evaluating travel chains as a whole. When travel chains are analysed, the whole chain should be considered, evaluating the public transportation service in itself is not enough. A travel chain consists of several elements, including public transportation services as well as walking and transfers. As an attempt to describe overall service quality, Kisgyörgy and Vasvári (2014) suggested the concept of Total Comfort Index (TCI) and Minimal TCI (minTCI) to describe the comfort conditions and their fluctuation along the travel chain. This paper aims to provide some tools to cover this gap in research and practice and to provide a methodology for this purpose.

The hypothesis of work if the comfort of travel chain is could be assessed. Therefore, the research question of this paper is if a framework could be built in order to assess the service quality along travel chains. The framework would extend the overall service quality concepts towards more accurate analysis and practical application. A simple fuzzy model could be used based on the overall indicators for information modelling and decision support. The proposed model is action-oriented, and identifies the weak points and the suggested improvements in the travel chain. The model is validated and its application is demonstrated through a real life case study in Budapest (Hungary).

\section{Methodology to determine the comfort of travel chain components}

\subsection{Attributes of travel comfort}

Availability determines whether or not transit service is an option for a particular trip, and when transit service is an option for a given trip only then is comfort and convenience considered by the passengers (TRB 2013). According to this, we evaluate these factors separately. In this paper we analyse the travel comfort features of travel chains. In this context, travel comfort is used in a broader sense to describe ride quality and transfer quality including mobility, information, safety, security, and naturally comfort aspects. It is not in the scope of this paper to develop a detailed methodology for the evaluation of the service quality attributes, for further reference TRB (2003) is suggested. The proposed methodology takes the attribute list as input: an arbitrary set of attributes can be included in the determination of service quality. Presenting the full attribute list used in this research is not possible in the frames of this paper, so we have included only some part of it for demonstration purposes. Table 1 shows one possible set of attributes for one element of the travel chain. The other parts of the journey have similar aspect and attribute structure, reflecting their characteristics.

The attributes of travel comfort, their evaluation and the relevant importance of their weights can be different from the point of view of different user groups, and they also depend on the local circumstances. So the comfort analysis should be done by relevant user groups, thus taking the human factor into account as well.

There are three methods to collect data: (1) statistical data, which can be measured objectively, (2) data evaluated by trained experts and (3) subjective data obtained through questionnaires. Subjective ratings measure the perception of passengers directly. However, it is biased by influencing subjective factors, which cannot be coupled directly to the service quality. On the other side, objective measures are useful, if they accurate, reliable and correlate to but does not depend on subjecting ratings (Strandemar 2005). Basically it is suggested to apply subjective and objective measures conjointly (Nathanail 2008; Friman, Fellesson 2009; Eboli, Mazzulla 2011; Hassan et al. 2013).

\subsection{Determination of Comfort Levels (CLs) of the attributes}

It is not within the scope of this paper to provide the exact definitions of the quality levels of attributes. Besides taking too much space, the definitions of quality levels greatly depends on the circumstances and perceptions of travellers, so this topic should be handled with a significant subjectivity. For a detailed guidance on how values might be determined or proper weights might be set, the Transit Cooperative Research Program (TCRP) Report 166 is suggested as a reference (Outwater et al. 2014).

Comfort Level (CL) is the indicator, which quantifies the utility value of attributes. A 5-point scale (with 
Table 1. One possible set of attributes of travel comfort at transfers, to demonstrate the aspect and attribute structure

\begin{tabular}{|c|c|c|}
\hline Aspect & Attribute & Description \\
\hline \multirow{8}{*}{ Access } & Distance of approach & Walking distance during the transfer \\
\hline & Time of approach & Walking time during the transfer \\
\hline & Directness of approach & Detours and waiting times \\
\hline & Grades & Level differences \\
\hline & Environment & $\begin{array}{l}\text { How attractive and encouraging the walking and waiting environment is, } \\
\text { including speedwalks, etc. }\end{array}$ \\
\hline & Protection against weather & How well protected the passenger against the weather during the transfer \\
\hline & Accessibility & Quality of access for people with disabilities \\
\hline & Access for service & How to pay fares \\
\hline \multirow{2}{*}{$\begin{array}{l}\text { Safety and } \\
\text { security }\end{array}$} & Security & $\begin{array}{l}\text { Objective and subjective security. Safety against crime, perceived security } \\
\text { and crime rates }\end{array}$ \\
\hline & Safety & Objective and subjective safety. Perceived safety and accident rates \\
\hline \multirow{4}{*}{ Waiting } & Connection availability & How frequent the service is, which passengers are transferring to \\
\hline & Delay & The delay relative to total travel time, caused by the transfer \\
\hline & Furniture & Availability of benches and shelters \\
\hline & Services & Availably services at the stops (e.g. shops) \\
\hline \multirow{3}{*}{ Information } & Wayfinding information & Information system helping to find the boarding location \\
\hline & Service information & Availability of schedule, route map and transfer routes \\
\hline & Real-time information & $\begin{array}{l}\text { Real time information about stops and transfers, expected arrival times. } \\
\text { Helping in unexpected traffic situation }\end{array}$ \\
\hline
\end{tabular}

categories "dissatisfactory", "low", "medium", "high" and "excellent") is used to categorize it. The CL and the importance of service quality attributes can be determined based on user surveys or on expert opinions. In both cases the users or the experts assign a CL and an $I_{i}$ importance rate to attribute $i$. The analytical hierarchy process and its numerous variants are proposed to determine the importance rates with a pair-wise comparison and to check the consistency of the importance rates (Markovits-Somogyi 2011; Duleba et al. 2013; Mukherjee 2014).

Human factors are very difficult to measure and quantify. Users' perceptions are heterogeneous, so by clustering users who share some common characteristic user opinions about the CLs can be homogenized. To create user groups in which the users have more or less homogeneous needs and perceptions of comfort and convenience, classification tree techniques are very suitable where the classification can be based on different market segmentations such as gender, age reason for travelling, etc. (De Oña et al. 2014; De Oña, R., De Oña, J. 2015). The analysis of comfort conditions should be done for all the relevant user groups, and their CLs should be determined respectively.

\subsection{Comfort of a component in the travel chain}

The Comfort Index (CI) is a measure, which describes the compound comfort of a component in the travel chain. The CI is determined as the sum of CLs of attributes multiplied by the importance weight of the attribute:

$$
C I=\sum_{i=1}^{n} C L_{i} \cdot w_{i},
$$

where: $C I$ - comfort index of a component in the travel chain, with values in the range $[1,5] ; C L_{i}$ - comfort level of the attribute $i$ of the given component; $w_{i}$-importance weight of the attribute $i$ of the given component; $n$ - number of attributes considered at the given component.

The perception of comfort is heavily influenced by the worst case. The Minimal Comfort Level (minCL), which is equal to the lowest CL of the component, was created to describe this worst case. Here the lowest CL of the component is defined as the lowest CL value where the cumulative weight belonging to this given CL value exceeds $10 \%$. This way the marginal annoyances can be omitted from the calculation.

$$
\begin{aligned}
& \min C L=\min \left(C L_{i} \mid \sum_{j=1}^{n} w_{j} \cdot \delta_{i, j} \geq 0.1\right), \\
& \delta_{i, j}= \begin{cases}1, & C L_{i} \geq C L_{j} ; \\
0, & C L_{i}<C L_{j},\end{cases}
\end{aligned}
$$

where: $\min C L$ - minimal comfort level of a component in the travel chain; $C L_{i}$ - comfort level of the attribute $i$ of the given component; $w_{i}$ - importance weight of the attribute $i$ of the given component; $n$ - number of attributes considered at the given component.

\section{Methodology to determine the comfort along the whole travel chain}

\subsection{Indices characterizing comfort}

To depict the qualitative characteristics of a certain travel chain the overall quality of the travel chain should be evaluated and the necessary actions should be determined. 
For this purpose, three indices are suggested: the TCI, the minimal TCI (minTCI) and the maximal TCI (maxTCI).

The TCI describes the overall quality along the travel chain. Based on this index travel chains can be compared to each other qualitatively. The other two indices are for estimating the homogeneity of the comfort conditions along the travel chain. They support decision making by identifying the service improvement priorities and the most effective improvement strategies.

\subsubsection{The TCI}

Travel comfort along the travel chain can be defined as the weighted average of the CI of the components in the travel chain, where the individual CIs are weighted by their expected travel time (Kisgyörgy, Vasvári 2014):

$$
\begin{aligned}
& T C I=\frac{1}{T T I} \cdot\left(\sum_{i=1}^{2} w t_{i} \cdot C I_{w, i}+\sum_{i=1}^{n} t t_{i} \cdot C I_{i}+\right. \\
& \left.\sum_{i=1}^{n-1} t r t_{i, i+1} \cdot\left(C I_{i, i+1}-0.5\right)\right),
\end{aligned}
$$

where: TCI - comfort index along the travel chain (TCI describes the average CL along the travel chain, and a 5 -point scale with categories "dissatisfactory", "low", "medium", "high" and "excellent" is used to categorize TCI); TTI - sum of expected travel times along the travel chain, called "travel time indicator"; $w t_{i}$ - the expected time spent by walking at the beginning $(i=1)$ and at the end $(i=2)$ of the travel; $t t_{i}$ - expected travel time of transportation mode or service $i$; $C I_{i}$ - the comfort index of transportation mode or service $i$; $\operatorname{trt}_{i, i+1}$ - expected transfer time between transportation modes $i$ and $i+1 ; C I_{i, i+1}-$ the comfort index of transfer between transportation modes $i$ and $i+1$ (the CI of transfer should reflect the discomfort of transfer compared to the direct transportation, so a 0.5 penalty term is applied).

\subsubsection{The minTCI}

The minTCI shows the worst element influenced perception of comfort in the travel chain, and is classified into the same 5-point scale as TCI (Kisgyörgy, Vasvári 2014). We modified the original definition of the index as the lowest minCI along the travel chain:

$$
\min T C I=\min \left(\min C I_{w, j}, \min C I_{i}, \min C I_{i, i+1}-0.5\right),
$$

where: $\min T C I$ - the minimal comfort index along the travel chain; $\operatorname{minCI} I_{w, j}$ - the minimal comfort index of walking at the beginning and at the end of the travel; min$\mathrm{CI}_{i}$ - the minimal Comfort Index of transportation mode or service $i$; $\operatorname{minCI}_{i, i+1}$ - the minimal comfort index of transfer between transportation modes $i$ and $i+1$ (the CI of transfer should reflect the discomfort of transfer compared to the direct transportation, so a 0.5 penalty term is applied).

\subsubsection{The maxTCI}

The maxTCI shows the best case influenced perception of comfort, and is classified into the same 5-point scale as TCI (Kisgyörgy, Vasvári 2014). We modified the original definition of the index as the highest maxCI along the travel chain:

$$
\max T C I=\max \left(C I_{w, j}, C I_{i}, C I_{i, i+1}-0.5\right),
$$

where: $\max T C I$ - the maximal Comfort Index along the travel chain; $C I_{w, j}$ - the comfort index of walking at the beginning and at the end of the travel; $C I_{i}$ - the Comfort Index of transportation mode or service $i$; $C I_{i, i+1}$ - the comfort index of transfer between transportation modes $i$ and $i+1$ (the CI of transfer should reflect the discomfort of transfer compared to the direct transportation, so a 0.5 penalty term is applied).

\subsection{Characteristics of comfort conditions}

The indices grab the characteristics features of the comfort conditions along the travel chain. Based on them the characteristics of comfort conditions along the travel chain can be classified into four different groups: homogenous, homogeneous with local problems, homogeneous with local excellences, and inhomogeneous (Figure 1).

The different characteristics require different improvement strategies. With homogeneous characteristics, if the TCI is too low, the CL of most important elements should be raised. However, maintaining homogeneity is also an issue, so the raise of the minimal CLs might be necessary. In case of inhomogeneous characteristics achieving homogeneity is the main task by improving the worst elements in the chain. In this methodology two actions are proposed to implement the improvement strategies: raising the CLs of characteristic elements (RAISECHAR), and raising the CLs of the worst elements (RAISEWORST), where RAISECHAR is to raise the general level of comfort along the travel chain and RAISEWORST is to homogenize the CLs along the travel chain.

Characteristic elements are the components in the travel chain with the largest weights in the index. In the vast majority of cases the characteristic elements are the public transport vehicles. So raising the CL of the characteristic element means the improvement of the travel comfort on the board of some or all of the vehicles. Different scenarios should be evaluated to find those transportation services whose improvement increases the most effectively and efficiently the CL of the whole travel chain. Worst elements mean those components of the travel chain whose $\mathrm{CL}$ is lower or equal to minTCI. The action of raising the CLs of the worst elements is defined as improving the CLs of all these elements. It should be noted that the improvement of the CL can be done in several ways; detailed analysis is needed to determine the most effective and efficient measures. 


\subsection{Fuzzy evaluation}

The above classification is not crisp; the transition between the groups is continuous, so building a fuzzy system on these indices makes it possible to describe the comfort conditions and to determine the necessary actions more accurately. With their ability to directly model such imprecise information, fuzzy systems reduce the overall cognitive dissonance in the modelling process. Fuzzy logic provides a method of reducing as well as explaining system complexity. In this view of modelling complex systems, the underlying mechanics are represented linguistically rather than mathematically.

Fuzzy systems consist of three main parts: linguistic variables, membership functions, and rules. Linguistic variables represent the input and output data. Linguistic terms represent the categories for the values of the linguistic variables, and membership function represents the degree of membership of linguistic variables within their linguistic terms. Finally, rules describe the relationship between input and output variables based on their linguistic terms (Cox 1998).

In the proposed methodology, the linguistic variables consist of the three indices (TCI, minTCI, maxTCI) as input variables and the actions (RAISECHAR, RAISEWORST) as output variables. The input variables lie in the interval $[1,5]$ by definition and are described by three linguistic terms. The output variables have three categories representing the necessary amount of level raise in comfort (Figure 2). Here the maximum raise of CLs is two levels, because more than that would be superfluous.

Table 2 shows the fuzzy classification of comfort characteristics; and the rules that determine the principal functionality of the system are summarized in Table 3. The rules together describe the actions needed to ensure an adequate and homogenous CL along the travel chain. a)

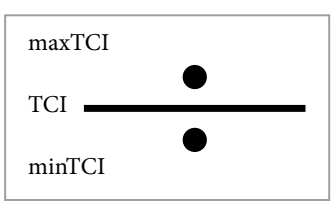

b)

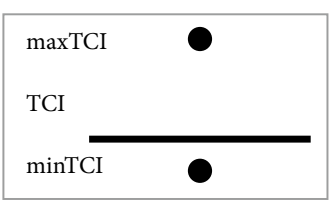

c)

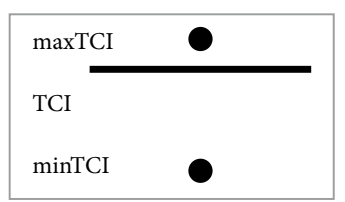

d)

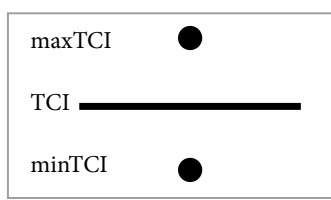

Figure 1. Comfort characteristics types: a - homogeneous; b - homogeneous with local excellences; $\mathrm{c}$ - homogeneous with local problems; $\mathrm{d}$ - inhomogeneous

a)

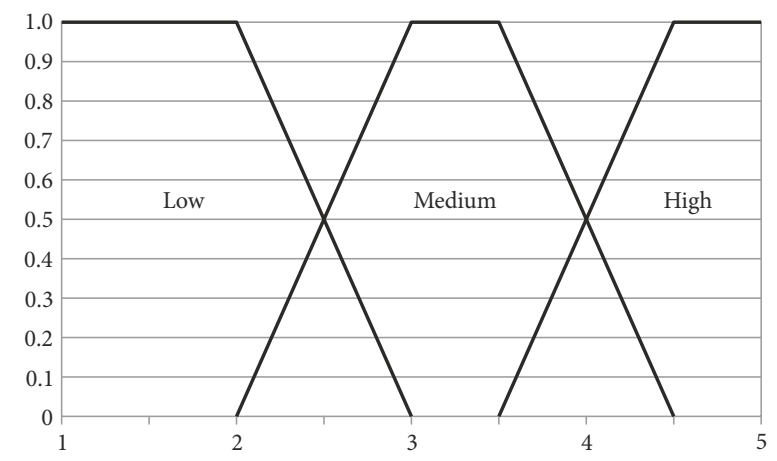

b)

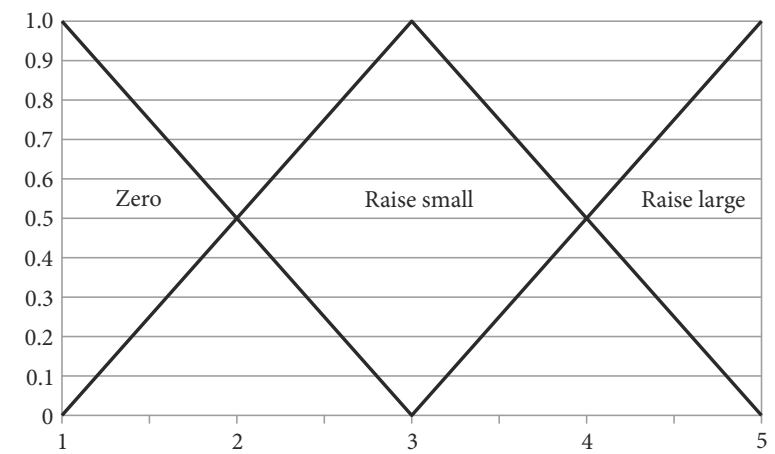

Figure 2. Linguistic terms and membership functions of the input (a) and output (b) linguistic variables

Table 2. Fuzzy classification for comfort characteristics

\begin{tabular}{|c|c|c|c|c|c|c|c|}
\hline \multirow{11}{*}{ IF } & TCI & \multirow{11}{*}{ AND } & $\operatorname{minTCI}$ & \multirow{11}{*}{ AND } & $\operatorname{maxTCI}$ & \multirow{11}{*}{ THEN } & Homogeneity class \\
\hline & \multirow{3}{*}{ low } & & low & & low & & homogeneous \\
\hline & & & low & & medium & & homogeneous \\
\hline & & & low & & high & & homogeneous with excellencies \\
\hline & \multirow{4}{*}{ medium } & & low & & medium & & homogeneous with problems \\
\hline & & & low & & high & & inhomogeneous \\
\hline & & & medium & & medium & & homogeneous \\
\hline & & & medium & & high & & homogeneous with excellencies \\
\hline & \multirow{3}{*}{ high } & & low & & high & & homogeneous with problems \\
\hline & & & medium & & high & & homogeneous \\
\hline & & & high & & high & & homogeneous \\
\hline
\end{tabular}


Table 3. Complete rule base for action

\begin{tabular}{|c|c|c|c|c|c|c|c|c|c|}
\hline \multirow{11}{*}{ IF } & TCI & \multirow{11}{*}{ AND } & $\operatorname{minTCI}$ & \multirow{11}{*}{ AND } & $\operatorname{maxTCI}$ & \multirow{11}{*}{ THEN } & RAISECHAR & \multirow{11}{*}{ AND } & RAISEWORST \\
\hline & \multirow{3}{*}{ low } & & low & & low & & raise large & & raise small \\
\hline & & & low & & medium & & raise large & & raise small \\
\hline & & & low & & high & & raise large & & raise small \\
\hline & \multirow{4}{*}{ medium } & & low & & medium & & raise small & & raise large \\
\hline & & & low & & high & & zero & & raise large \\
\hline & & & medium & & medium & & raise small & & raise small \\
\hline & & & medium & & high & & raise small & & zero \\
\hline & \multirow{3}{*}{ high } & & low & & high & & zero & & raise large \\
\hline & & & medium & & high & & zero & & raise small \\
\hline & & & high & & high & & zero & & zero \\
\hline
\end{tabular}

Table 4. Characteristics of the analysed travel chains

\begin{tabular}{|c|c|c|c|c|c|c|c|c|}
\hline \multirow{3}{*}{$\begin{array}{c}\text { Description } \\
\text { Walking at the beginning }\end{array}$} & \multirow{2}{*}{\multicolumn{2}{|c|}{$\begin{array}{c}\text { Travel Chain } 1 \\
\text { Bus }\end{array}$}} & \multirow{2}{*}{\multicolumn{2}{|c|}{$\begin{array}{c}\text { Travel Chain } 2 \\
\text { Subway }\end{array}$}} & \multirow{2}{*}{\multicolumn{2}{|c|}{$\begin{array}{l}\text { Travel Chain } 3 \\
\text { Bus + subway }\end{array}$}} & \multirow{2}{*}{\multicolumn{2}{|c|}{$\frac{\text { Travel Chain } 4}{\text { Tram + subway }}$}} \\
\hline & & & & & & & & \\
\hline & & $2 \mathrm{~min}$ & & $4 \mathrm{~min}$ & & $2 \mathrm{~min}$ & & $2 \mathrm{~min}$ \\
\hline Bus & $9 \mathrm{~km}$ & $33 \mathrm{~min}$ & & & $6.5 \mathrm{~km}$ & $22 \mathrm{~min}$ & & \\
\hline Tram & & & & & & & $6.5 \mathrm{~km}$ & $18 \mathrm{~min}$ \\
\hline Metro 4 & & & $9 \mathrm{~km}$ & $14 \mathrm{~min}$ & $2.5 \mathrm{~km}$ & $5 \mathrm{~min}$ & $2.5 \mathrm{~km}$ & $5 \mathrm{~min}$ \\
\hline Transfer & & & & & & $5 \mathrm{~min}$ & & $5 \mathrm{~min}$ \\
\hline Walking at the end & & $2 \min$ & & $4 \mathrm{~min}$ & & $2 \min$ & & $2 \min$ \\
\hline Sum & $9 \mathrm{~km}$ & $37 \mathrm{~min}$ & $9 \mathrm{~km}$ & $22 \mathrm{~min}$ & $9 \mathrm{~km}$ & $36 \mathrm{~min}$ & $9 \mathrm{~km}$ & $32 \mathrm{~min}$ \\
\hline
\end{tabular}

The evaluation of the model propositions is handled through an aggregation process that produces the final fuzzy regions for each output variable. In this methodology the mean of maximum defuzzification method is applied to decompose the solution region, which finds the domain point with the maximum truth.

\section{Application of methodology}

\subsection{Location}

We show the applicability of the comfort indicators and the fuzzy system by using an illustrative case study as an example. In the case study, we analyse the comfort of travel chains in one of the most loaded transport corridors of Budapest, capital of Hungary. In the corridor approximately $180000 \ldots 200000$ trips are made daily by public transport, the length of it is $9 \mathrm{~km}$.

A few years ago a bus service was solely available in the corridor, with excusive lanes and very high-frequency (30 bus/h), but the service was still sensitive to disturbances. The travel time varied between 26 and 33 min (Travel Chain 1).

The local government of Budapest decided to build a new subway line, Metro 4 (BKK 2018), along the corridor to improve the quality of public transportation (Travel Chain 2). However, the subway line, which was opened in 2014, was built only partially in the corridor, and in a $6.5 \mathrm{~km}$ long section still buses offer public transportation
(Travel Chain 3). According to the current development plans, a new tramway will cover the remaining length of the corridor (Travel Chain 4).

\subsection{Data}

In this case study, we analyse the effects of developments from the point of view of comfort.

The characteristics of the travel chains in peak hours are reported in Table 4. Transfer time includes the time needed to transfer between modes, and to access the subway at the beginning and the end of the travel chain. It can be seen that only the subway line built along the entire corridor means significant improvement in travel times, the other travel times are close to each other. We assumed 2 min walking at the beginning and at the end of the travel chains except the subway (4 min).

We used subjective rating to evaluate the attributes. Altogether 500 passengers who travelled along the analysed travel chain in the morning rush hours were interviewed about their perception of comfort. The participants were asked to evaluate both the level and the importance of the attributes of the elements along the analysed travel chain. Instead of the currently non-existing option of tram in Travel Chain 4, the questionnaire asked the opinion about the comfort attributes of a tram service in general, and these values were used to evaluate the hypothetical option. It is assumed in case of Travel Chain 2. 
Table 5. Comfort indices (CI, minCI, TCI, minTCI, maxTCI) of the analysed travel chains

\begin{tabular}{|c|c|c|c|c|c|c|c|c|}
\hline \multirow{3}{*}{ Description } & \multirow{2}{*}{\multicolumn{2}{|c|}{$\frac{\text { Travel Chain } 1}{\text { Bus }}$}} & \multirow{2}{*}{\multicolumn{2}{|c|}{$\frac{\text { Travel Chain } 2}{\text { Subway }}$}} & \multirow{2}{*}{\multicolumn{2}{|c|}{$\begin{array}{c}\text { Travel Chain } 3 \\
\text { Bus + subway }\end{array}$}} & \multirow{2}{*}{\multicolumn{2}{|c|}{$\begin{array}{c}\text { Travel Chain } 4 \\
\text { Tram + subway }\end{array}$}} \\
\hline & & & & & & & & \\
\hline & $\mathrm{CI}$ & $\min C I$ & $\mathrm{CI}$ & $\operatorname{minCI}$ & $\mathrm{CI}$ & $\min C I$ & $\mathrm{CI}$ & $\operatorname{minCI}$ \\
\hline Walking at the beginning & 4.05 & 3.27 & 4.41 & 4.03 & 4.05 & 3.27 & 4.17 & 3.36 \\
\hline Bus & 3.03 & 2.13 & & & 3.03 & 2.13 & & \\
\hline Tram & & & & & & & 4.13 & 3.34 \\
\hline Metro 4 & & & 4.62 & 4.08 & 4.62 & 4.08 & 4.62 & 4.08 \\
\hline Transfer $^{*}$ & & & & & $3.58^{*}$ & $2.43^{*}$ & $3.58^{*}$ & $2.43^{*}$ \\
\hline Walking at the end & 4.27 & 3.83 & 4.02 & 3.82 & 4.02 & 3.82 & 4.02 & 3.82 \\
\hline TCI & & 3.15 & & 4.47 & & 3.44 & & 4.12 \\
\hline $\operatorname{minTCI}$ & & 2.13 & & 4.08 & & 2.13 & & $2.43^{\star}$ \\
\hline $\operatorname{maxTCI}$ & & 4.27 & & 4.62 & & 4.62 & & 4.62 \\
\hline
\end{tabular}

Note: ${ }^{*}$ - a 0.5 penalty is applied.

These 500 passengers then were classified into homogeneous groups by the classification tree technique. The largest group had 387 members, basically commuters on the corridor; and we evaluated the travel chain based on the opinion of these commuters. Pair-wise comparison was used to determine the importance $I$ of attributes by the different aspects. The sum of $I$ values by aspects is 1 . The CLs of attributes were calculated as the mean of the opinions in the surveys. The CIs of aspects are the sum of attributes CIs weighted by the importance rates. We calculated the CIs of the components as the sum of aspect CIs weighted by expert judgment. Table 5 summarizes the data and the calculation, and shows the values of the indices TCI, minTCI and maxTCI. The fuzzy evaluation of the travel chains with the suggested actions is summarized are summarized in Table 6. Here each row displays the relevant rules from the rule base (Table 3 ) for each scenario, the cells showing the linguistic variables and their degree of membership, based on the definitions in Figure 2 and the results in Table 1.

\subsection{Results}

The results clearly agree with common sense. The comfort along Travel Chain 1 is homogeneous with local excellences. The index of minTCI shows the problems of the bus service: the overcrowding and the dynamic ride quality. The TCI value states that the overall comfort of the travel chain is rated medium. The fuzzy evaluation of the indices indicates the most effective intervention, which is improving the characteristic and the worst elements along the chain simultaneously, which means the upgrade of the comfort on the bus, especially dynamic ride quality and overcrowding.

The original development plans, building a subway line all along the corridor would have tackled these problems. The indices of Travel Chain 2 reflect the significant improvement in comfort (rated very good), and indicate the importance of quality of access to the subway, because that will be the most uncomfortable element of the chain.
However, this chain has a high comfort; there is no need to do anything.

In case of the current situation, Travel Chain 3, the increase in comfort due to the subway line is lost at the transfer. In this chain the new subway line only increased the inhomogeneity of the chain with the additional discomfort of the transfer, and the ride conditions on the bus still remains an issue. The new subway line slightly improved the overall quality of the travel chain, close to Travel Chain 1, but clearly did not solved the comfort problems. This fact is in accordance with the results of the methodology, which show that instead of the raise of the average comfort the worst elements in the chain should have been improved. In addition, the experiences confirm this result: the majority of people does not transfer to the subway line but go on by bus.

Building the tramway, the intended development in the corridor has better results. The tram has higher capacity and smoother run, so it overcomes the biggest comfort problems of bus service; it has a good comfort category. Here the transfer becomes the major discomfort. Therefore, to provide a smooth CL along the corridor the transfer should be as easy and convenient as possible.

Park and Ride $(P+R)$ has a low weight at the access of the public transportation services. The reason is that the corridor serves mainly the residents of Ujpalota (a district in Budapest), shifting from passenger car to public transport is minimal. However, if the goal is to encourage modal shift, then $\mathrm{P}+\mathrm{R}$ should receive a much higher weight. In this case, its effect will appear in the comfort indices, calling the attention to the necessary interventions to improve $\mathrm{P}+\mathrm{R}$ access to the public transport service.

\section{Discussion}

Our results show that the proposed methodology for evaluate travel comfort along the travel chain gives a good description of the overall characteristics of comfort, can identify the problems and can determine the components whose improvement can the most effectively enhance the 
Table 6. Complete evaluation based on the fuzzy rules

\begin{tabular}{|c|c|c|c|c|c|c|c|c|c|}
\hline \multicolumn{10}{|c|}{ Travel Chain 1} \\
\hline \multirow{5}{*}{ IF } & TCI & \multirow{5}{*}{ AND } & $\operatorname{minTCI}$ & \multirow{5}{*}{ AND } & $\operatorname{maxTCI}$ & \multirow{5}{*}{ THEN } & RAISECHAR & \multirow{5}{*}{ AND } & RAISEWORST \\
\hline & \multirow{4}{*}{ M 1.00} & & L 0.87 & & M 0.23 & & $\mu($ raise small $)=0.23$ & & $\mu($ raise large $)=0.23$ \\
\hline & & & L 0.87 & & Н 0.77 & & $\mu($ zero $)=0.77$ & & $\mu($ raise large $)=0.77$ \\
\hline & & & M 0.13 & & M 0.23 & & $\mu($ raise small $)=0.13$ & & $\mu($ raise small $)=0.13$ \\
\hline & & & M 0.13 & & Н 0.77 & & $\mu($ raise small $)=0.13$ & & $\mu($ zero $)=0.13$ \\
\hline \multicolumn{10}{|c|}{ Travel Chain 2} \\
\hline \multirow{4}{*}{ IF } & TCI & \multirow{4}{*}{ AND } & $\operatorname{minTCI}$ & \multirow{4}{*}{ AND } & $\operatorname{maxTCI}$ & \multirow{4}{*}{ THEN } & RAISECHAR & \multirow{4}{*}{ AND } & RAISEWORST \\
\hline & M 0.03 & & M 0.42 & & H 1.00 & & $\mu($ raise small $)=0.03$ & & $\mu($ zero $)=0.03$ \\
\hline & \multirow{2}{*}{ Н 0.97} & & M 0.42 & & H 1.00 & & $\mu($ zero $)=0.42$ & & $\mu($ raise small $)=0.42$ \\
\hline & & & H 0.58 & & H 1.00 & & $\mu($ zero $)=0.58$ & & $\mu($ zero $)=0.58$ \\
\hline \multicolumn{10}{|c|}{ Travel Chain 3} \\
\hline \multirow{3}{*}{ IF } & TCI & \multirow{3}{*}{ AND } & $\operatorname{minTCI}$ & \multirow{3}{*}{ AND } & $\operatorname{maxTCI}$ & \multirow{3}{*}{ THEN } & RAISECHAR & \multirow{3}{*}{ AND } & RAISEWORST \\
\hline & \multirow{2}{*}{ M 1.00} & & L 0.87 & & H 1.00 & & $\mu($ zero $)=0.87$ & & $\mu($ raise large $)=0.87$ \\
\hline & & & M 0.13 & & H 1.00 & & $\mu($ raise small $)=0.13$ & & $\mu($ zero $)=0.13$ \\
\hline \multicolumn{10}{|c|}{ Travel Chain 4} \\
\hline \multirow{5}{*}{ IF } & TCI & \multirow{5}{*}{ AND } & $\operatorname{minTCI}$ & \multirow{5}{*}{ AND } & $\max T C I$ & \multirow{5}{*}{ THEN } & RAISECHAR & \multirow{5}{*}{ AND } & RAISEWORST \\
\hline & $M 038$ & & L 0.57 & & H 1.00 & & $\mu($ zero $)=0.38$ & & $\mu($ raise large $)=0.38$ \\
\hline & 110.38 & & M 0.43 & & H 1.00 & & $\mu($ raise small $)=0.38$ & & $\mu($ zero $)=0.38$ \\
\hline & \multirow{2}{*}{ H 0.62} & & L 0.57 & & H 1.00 & & $\mu($ zero $)=0.57$ & & $\mu($ raise large $)=0.57$ \\
\hline & & & M 0.43 & & H 1.00 & & $\mu($ zero $)=0.43$ & & $\mu($ raise small $)=0.43$ \\
\hline
\end{tabular}

Notes:

- L - low, M - medium, H - high;

- the numbers show the degree of membership for each linguistic variable;

- the outputs with the highest degree of membership are indicated with bold letters for each scenario.

overall quality along the travel chain. The overall travel comfort influences very heavily the competitiveness of a travel chain, so its analysis helps to determine the most efficient and effective actions to make a chain more attractive and competitive. In addition, the methodology makes it possible to compare the comfort of different travel chains and to evaluate the effects of interventions as well.

In this paper we have suggested a fuzzy approach to evaluate the quality of comfort along a travel chain, where comfort is used in the broad sense to describe overall service quality. The travel chains include all components of a trip, such as the walking at the beginning and at the end, riding public transportation vehicles, and the transfers between them. The comfort of these components can be described by several ways; here in this methodology we proposed one type of index, the CI. The characteristics of the overall comfort along the travel chain are featured by three indices, the TCI, the minTCI and the maxTCI. The first of these indices characterizes the integrated comfort conditions along the travel chain and creates the basis of comparison between different travel chains, while the minimal and maximal indices describe the inhomogeneity characteristics.

The evaluation of comfort along the travel chain involves subjective and imprecise assessments, which are of fuzzy nature. Fuzzy assessments expressed in linguistic terms are often the most intuitive and effective way to evaluate these problems. In this paper, we have presented an effective fuzzy approach to identify the most effective and efficient actions for homogenizing and improving travel comfort along the travel chain. The proposed methodology provides a tool to easily and simply identify the most effective measures to promote modal shift toward sustainable transportation modes and to evaluate the expectable effect of intended measures. Before the expensive investment, it can be identified, whether it will bring about an improvement in comfort. It can be stated, that a successful development has a one category better TCI, than that of the previously available service.

An empirical study was carried out to exemplify the approach, where the validity and applicability of the method was tested as well. The case study was conducted for one of the major travel corridors of Budapest (Hungary). In this corridor, public transportation was significantly improved in the recent past, and data for evaluating comfort conditions both before and after the improvement could be collected. Detailed engineering analysis and travel behaviour of users confirmed the results of the methodology, regarding both the characteristics and the necessary actions. Therefore, the methodology describes the overall 
conditions well and points out the weak parts. By the application of the methodology very valuable insights can be gained regarding the location and type of the necessary interventions.

The results of our research are helpful to evaluate the actual service level of sustainable alternatives of individual car usage and to promote modal shift towards sustainable transportation modes. However, it would be useful to extend the fuzzy approach further; the application of fuzzy weights to determine the comfort indices of the individual components in the travel chain is suggested for research in the future. In addition, the extension of the fuzzy approach to the comparison of the comfort along travel chains could be a future research topic.

\section{References}

BKK. 2018. Maps. Budapesti Közlekedési Központ (BKK) - Centre for Budapest Transport, Hungary. Available from Internet: https://bkk.hu/en/maps

Cascetta, E.; Cartenì, A. 2014. The hedonic value of railways terminals. A quantitative analysis of the impact of stations quality on travellers behaviour, Transportation Research Part A: Policy and Practice 61: 41-52.

https://doi.org/10.1016/j.tra.2013.12.008

Cherry, T.; Townsend, C. 2012. Assessment of potential improvements to metro-bus transfers in Bangkok, Thailand, Transportation Research Record: Journal of the Transportation Research Board 2276: 116-122. https://doi.org/10.3141/2276-14

Cox, E. 1998. The Fuzzy Systems Handbook: a Practitioner's Guide to Building, Using, and Maintaining Fuzzy Systems. AP Professional. $716 \mathrm{p}$.

De Abreu e Silva, J.; Bazrafshan, H. 2013. User satisfaction of intermodal transfer facilities in Lisbon, Portugal, Transportation Research Record: Journal of the Transportation Research Board 2350: 102-110. https://doi.org/10.3141/2350-12

De Oña, J.; De Oña, R.; Eboli, L.; Mazzulla, G. 2013. Perceived service quality in bus transit service: a structural equation approach, Transport Policy 29: 219-226. https://doi.org/10.1016/j.tranpol.2013.07.001

De Oña, R.; De Oña, J. 2015. Analysis of transit quality of service through segmentation and classification tree techniques, Transportmetrica A: Transport Science 11(5): 365-387. https://doi.org/10.1080/23249935.2014.1003111

De Oña, R.; Eboli, L.; Mazzulla, G. 2014. Key factors affecting rail service quality in the Northern Italy: a decision tree approach, Transport 29(1): 75-83.

https://doi.org/10.3846/16484142.2014.898216

Dell'Olio, L.; Ibeas, A.; Cecín, P.; Dell'Olio, F. 2011. Willingness to pay for improving service quality in a multimodal area, Transportation Research Part C: Emerging Technologies 19(6): 1060-1070. https://doi.org/10.1016/j.trc.2011.06.004

Duleba, S.; Shimazaki, Y.; Mishina, T. 2013. An analysis on the connections of factors in a public transport system by AHPISM, Transport 28(4): 404-412.

https://doi.org/10.3846/16484142.2013.867282

Eboli, L.; Mazzulla, G. 2011. A methodology for evaluating transit service quality based on subjective and objective measures from the passenger's point of view, Transport Policy 18(1): 172-181. https://doi.org/10.1016/j.tranpol.2010.07.007
Eboli, L.; Mazzulla, G. 2009. A new customer satisfaction index for evaluating transit service quality, Journal of Public Transportation 12(3): 21-37.

https://doi.org/10.5038/2375-0901.12.3.2

Eboli, L.; Mazzulla, G. 2015. Relationships between rail passengers' satisfaction and service quality: a framework for identifying key service factors, Public Transport 7(2): 185-201. https://doi.org/10.1007/s12469-014-0096-x

Eboli, L.; Mazzulla, G. 2007. Service quality attributes affecting customer satisfaction for bus transit, Journal of Public Transportation 10(3): 21-34. https://doi.org/10.5038/2375-0901.10.3.2

Friman, M.; Fellesson, M. 2009. Service supply and customer satisfaction in public transportation: the quality paradox, Journal of Public Transportation 12(4): 57-69. https://doi.org/10.5038/2375-0901.12.4.4

Guihaire, V.; Hao, J.-K. 2008. Transit network design and scheduling: a global review, Transportation Research Part A: Policy and Practice 42(10): 1251-1273.

https://doi.org/10.1016/j.tra.2008.03.011

Hassan, M. N.; Hawas, Y. E.; Ahmed, K. 2013. A multi-dimensional framework for evaluating the transit service performance, Transportation Research Part A: Policy and Practice 50: 47-61. https://doi.org/10.1016/j.tra.2013.01.041

Hernandez, S.; Monzon, A.; De Oña, R. 2016. Urban transport interchanges: a methodology for evaluating perceived quality, Transportation Research Part A: Policy and Practice 84: 31-43. https://doi.org/10.1016/j.tra.2015.08.008

Iseki, H.; Taylor, B. D. 2010. Style versus service? An analysis of user perceptions of transit stops and stations, Journal of Public Transportation 13(3): 23-48. https://doi.org/10.5038/2375-0901.13.3.2

Kisgyörgy, L.; Vasvári, G. 2014. Travel chain based urban mobility, in Second International Conference on Traffic and Transport Engineering (ICTTE), 27-28 November 2014, Belgrade, Serbia, 69-75.

López-Lambas, M. E.; Monzon, A. 2010. Private funding and management for public interchanges in Madrid, Research in Transportation Economics 29(1): 323-328.

https://doi.org/10.1016/j.retrec.2010.07.041

Markovits-Somogyi, R. 2011. Modification of a DEA-AHP based method for ranking the decision making units, in 9th International Conference on Data Envelopment Analysis (DEA2011), 25-27 August 2011, Thessaloniki, Greece.

Mukherjee, K. 2014. Analytic hierarchy process and technique for order preference by similarity to ideal solution: a bibliometric analysis 'from' past, present and future of AHP and TOPSIS, International Journal of Intelligent Engineering Informatics 2(2/3): 96-117. https://doi.org/10.1504/IJIEI.2014.066210

Nathanail, E. 2008. Measuring the quality of service for passengers on the Hellenic railways, Transportation Research Part A: Policy and Practice 42(1): 48-66. https://doi.org/10.1016/j.tra.2007.06.006

Outwater, M.; Sana, B.; Ferdous, N.; Woodford, B.; Lobb, J.; Schmitt, D.; Roux, J.; Bhat, C.; Sidharthan, R.; Sidharthan, R.; Hess, S. 2014. Characteristics of Premium Transit Services that Affect Choice of Mode. Transit Cooperative Research Program (TCRP) Report 166. Transportation Research Board (TRB), Washington, DC, US. 394 p. https://doi.org/10.17226/22401 
Strandemar, K. 2005. On Objective Measures for Ride Comfort Evaluation. Royal Institute of Technology (KTH), Stockholm, Sweden. 99 p. Available from Internet:

http://kth.diva-portal.org/smash/get/diva2:14199/FULLTEXT01.pdf

TRB. 2003. A Guidebook for Developing a Transit PerformanceMeasurement System. Transit Cooperative Research Program (TCRP) Report 88. Transportation Research Board (TRB), Washington, DC, US. 383 p. Available from Internet: http://onlinepubs.trb.org/onlinepubs/tcrp/tcrp_rpt_88.pdf

TRB. 2013. Transit Capacity and Quality of Service Manual. Transit Cooperative Research Program (TCRP) Report 165. Transportation Research Board (TRB), Washington, DC, US. 805 p. https://doi.org/10.17226/24766

Tsalis, P.; Naniopoulos, A. 2012. Accessibility management at municipal level for people with restricted mobility: the case of Thessaloniki, Procedia - Social and Behavioral Sciences 48: 2597-2606. https://doi.org/10.1016/j.sbspro.2012.06.1230

Tyrinopoulos, Y.; Antoniou, C. 2008. Public transit user satisfaction: variability and policy implications, Transport Policy 15(4): 260-272. https://doi.org/10.1016/j.tranpol.2008.06.002 